\title{
Exciton Gating and Triplet Deshelving in Single Dye Molecules Excited by Perovskite Nanocrystal FRET Antennae
}

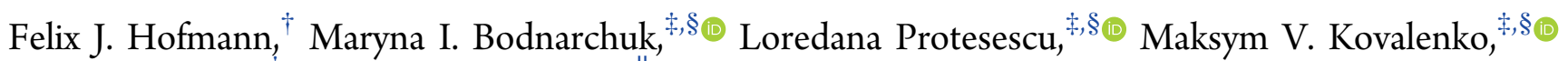 \\ John M. Lupton, ${ }^{\dagger}{ }^{\dagger}$ and Jan Vogelsang ${ }^{*}, \|_{\odot}$ \\ ${ }^{\dagger}$ Institut für Experimentelle und Angewandte Physik, Universität Regensburg, Universitätsstraße 31, 93053 Regensburg, Germany \\ ${ }^{\ddagger}$ Department of Chemistry and Applied Biosciences, ETH Zürich, Vladimir Prelog Weg 1, CH-8093 Zürich, Switzerland \\ ${ }^{\S}$ Empa - Swiss Federal Laboratories for Materials Science and Technology, Überlandstrasse 129, CH-8600 Dübendorf, Switzerland \\ "Department Chemie, Ludwig-Maximilians-Universität München, Butenandtstrasse 5-13, 81377 München, Germany
}

\section{Supporting Information}

ABSTRACT: The extraordinary absorption cross section and high photoluminescence (PL) quantum yield of perovskite nanocrystals make this type of material attractive to a variety of applications in optoelectronics. For the same reasons, nanocrystals are also ideally suited to function as nanoantennae to excite nearby single dye molecules by fluorescence resonance energy transfer (FRET). Here, we demonstrate that $\mathrm{FAPbBr}_{3}$ perovskite nanocrystals, of cuboidal shape and approximately $10 \mathrm{~nm}$ in size, are capable of selectively exciting single cyanine 3 molecules at a concentration 100-fold higher than standard single-molecule concentrations. This FRET antenna mechanism increases the effective brightness of the single dye molecules 100 -fold. Photon statistics and emission polarization measurements provide evidence for the FRET process by revealing photon antibunching with unprecedented fidelity and highly polarized emission stemming from single dye molecules. Remarkably, the quality of single-photon emission improves 1.5 -fold compared to emission collected directly from the nanocrystals because the higher excited states of the dye molecule act as effective filters to multiexcitons. The same process gives rise to efficient deshelving of the molecular triplet state by reverse intersystem crossing (RISC), translating into a reduction of the PL saturation of the dye, thereby increasing the maximum achievable PL intensity of the dye by a factor of 3 .

$\mathrm{E}$ nhancing the fluorescence of single nanoscale objects, particularly of organic dye molecules, has long been the goal of nanophotonics. ${ }^{1-3}$ The advantages of bright nanoscale emitters are quite obvious. For example, lower excitation intensities can be used, leading to decreased background signals and reduced photodamage ${ }^{4,5}$ expensive photodetectors with high quantum detection efficiencies can be avoided, and fast dynamic processes, such as the folding mechanisms of proteins, can be followed with high time resolution. ${ }^{6,7}$ Two basic principles have been developed to successfully increase the fluorescence of single molecules by several orders of magnitude: (i) fluorescence enhancement by exploiting a plasmonic hotspot or using an optical antenna based on a metallic nanostructure ${ }^{1-3,8}$ and (ii) fluorescence enhancement by light-harvesting and efficient energy transfer. ${ }^{9-15}$ The first approach increases both the excitation and radiative rates, whereas the second approach raises only the excitation rate. For this reason, plasmonic fluorescence enhancement has the advantage that excited-state saturation of a dye molecule decreases significantly, and intersystem crossing into a dark triplet state becomes negligible because radiative relaxation occurs faster than the spin-flip. ${ }^{16,17}$ However, it is, to date, not possible to build freely diffusing fluorescent probes based on plasmonic enhancement to label biologically relevant struc-

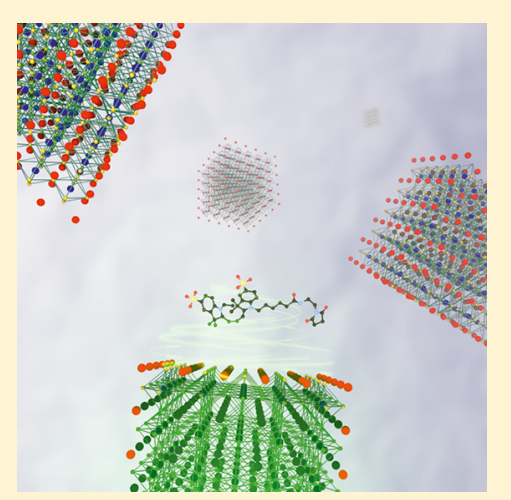

tures. Additionally, it is difficult to address single dye molecules within an ensemble due to the nonspecific enhancement of all dye molecules in the visible and IR wavelength regions. ${ }^{18}$ For these reasons, fluorescence enhancement by light-harvesting rather than plasmonic antennae is worthwhile pursuing. This approach was demonstrated on a variety of different fluorescence resonance energy transfer (FRET) assemblies, e.g., in conjugated polymer nanoparticles, ${ }^{12,13,19}$ semiconductor dye assemblies, ${ }^{20-24}$ porphyrin arrays, ${ }^{15}$ micellar nanoparticles, ${ }^{25}$ and DNA. ${ }^{26}$ Very recently, a new benchmark was set by Trofymchuk et al. with polymer nanoparticles containing more than 10000 donor dyes, which funnel their energy toward one single acceptor dye and therefore enhance the dye's fluorescence 1000 -fold. ${ }^{11}$ This strong enhancement of light absorption enabled single-particle microscopy under excitation powers equivalent to sunlight, ${ }^{11}$ but strong saturation of the acceptor dye generally prohibits strong fluorescence enhancement under high excitation intensities. We conclude, therefore, that three aspects must be combined to achieve very bright single-particle fluorescence: (i) a large

Received: January 21, 2019

Accepted: January 28, 2019

Published: February 21, 2019 
absorption cross section of the absorbing unit, (ii) efficient excitation energy transfer, and (iii) nonsaturable acceptor dyes.

We tackle this challenge by using formamidinium lead bromide $\left(\mathrm{FAPbBr}_{3}\right)$ perovskite nanocrystals (NCs) as FRET antennae to drive the fluorescence of single organic dye molecules such as cyanine 3 (Cy3). Surprisingly, this donoracceptor FRET pair leads to deshelving of the triplet state in the dye, which is the dominant state responsible for the strong saturation of PL under intense excitation conditions. ${ }^{29}$ Detailed analysis of the fluorescence photon statistics suggests that higher excited states of the dye are responsible for this deshelving, which also enables gating of the multiexcitons formed in the perovskite NC.

Suitable $\mathrm{FAPbBr}_{3}$ NCs were synthesized by slightly modifying a published procedure, ${ }^{28}$ wherein the photoluminescence $(\mathrm{PL})$ could be tuned in the range of 470-535 $\mathrm{nm}$ by adjusting the NC size between 5 and $12 \mathrm{~nm}^{28}$ In addition, these NCs exhibit a high PL quantum yield of up to $85 \%$ and a narrow PL emission spectrum with a full width at half-maximum of $<22 \mathrm{~nm}^{28}$ These properties make such NCs excellent candidates as light-harvesting units and donors in FRET pairs. We chose cuboidal NCs with a mean edge length of approximately $10 \mathrm{~nm}$ because at this size the PL exhibits a strong overlap with the absorption spectrum of $\mathrm{Cy} 3$ and the NC and dye PL peaks can be well separated. Figure la shows a scanning transmission electron microscopy (STEM) image of such NCs. The absorption (dashed green and orange curves) and PL spectra (solid green and red curves) are shown in Figure $1 \mathrm{~b}$ for the NCs and Cy3, respectively, dispersed in a 100 $\mathrm{nm}$ thick poly(methyl-methacrylate) (PMMA) film by spincoating a toluene solution containing PMMA, NCs, and dyes on top of a cleaned glass cover slide. ${ }^{29}$ The concentration of the NCs and dyes was tuned so that approximately $1 \mathrm{NC}$ and 1000 dye molecules were present in a $2 \times 2 \times 0.1 \mu \mathrm{m}^{3}$ volume, as schematically depicted in Figure 1c. For this reason, the probability to find a single molecule inside of a $10 \times 10 \times 10$ $\mathrm{nm}^{3}$ cube, which constitutes a reasonable range for significant FRET to occur, is smaller than $1 \%$. Such a low probability ensures that energy transfer to multiple dyes or between dyes, i.e., HOMO-FRET, ${ }^{29-31}$ can be neglected. However, the same considerations lead to an estimate of exciting 10-20 dyes inside of a diffraction-limited excitation spot when exciting the dyes directly and not by FRET. The sample was scanned with a confocal microscope, exciting at $405 \mathrm{~nm}$ with $0.1 \mathrm{~kW} / \mathrm{cm}^{2}$ and collecting PL above $409 \mathrm{~nm}$ to obtain an image as shown in Figure $1 \mathrm{~d}^{29}$ Spectra were recorded from diffraction-limited spots, and an example is shown in Figure 1e. The black curve displays a typical spectrum for early recording times $(\sim 0-4 \mathrm{~s}$ after initial laser exposure), and the green curve is for late recording times $(\sim 15-19$ s). Comparison with the emission spectra in Figure $1 \mathrm{~b}$ reveals that the spectrum at early recording times has perfect overlap with both the $\mathrm{NC}$ and dye emission spectra. At later recording times, the dye emission component vanishes due to photobleaching. Direct excitation of the dye at $405 \mathrm{~nm}$ is not possible given the lack of absorption; hence, efficient energy transfer must occur from NCs to dyes. The spectral detection windows indicated in Figure 1e (green $409-530 \mathrm{~nm}$ and red $600-750 \mathrm{~nm}$ ) are used in the following experiments to differentiate between emission stemming from the NCs and from the dyes. Figure if shows a typical PL intensity transient recorded with such spectral detection windows from a single NC-dye FRET pair excited at $405 \mathrm{~nm}$. More examples are shown in the Supporting
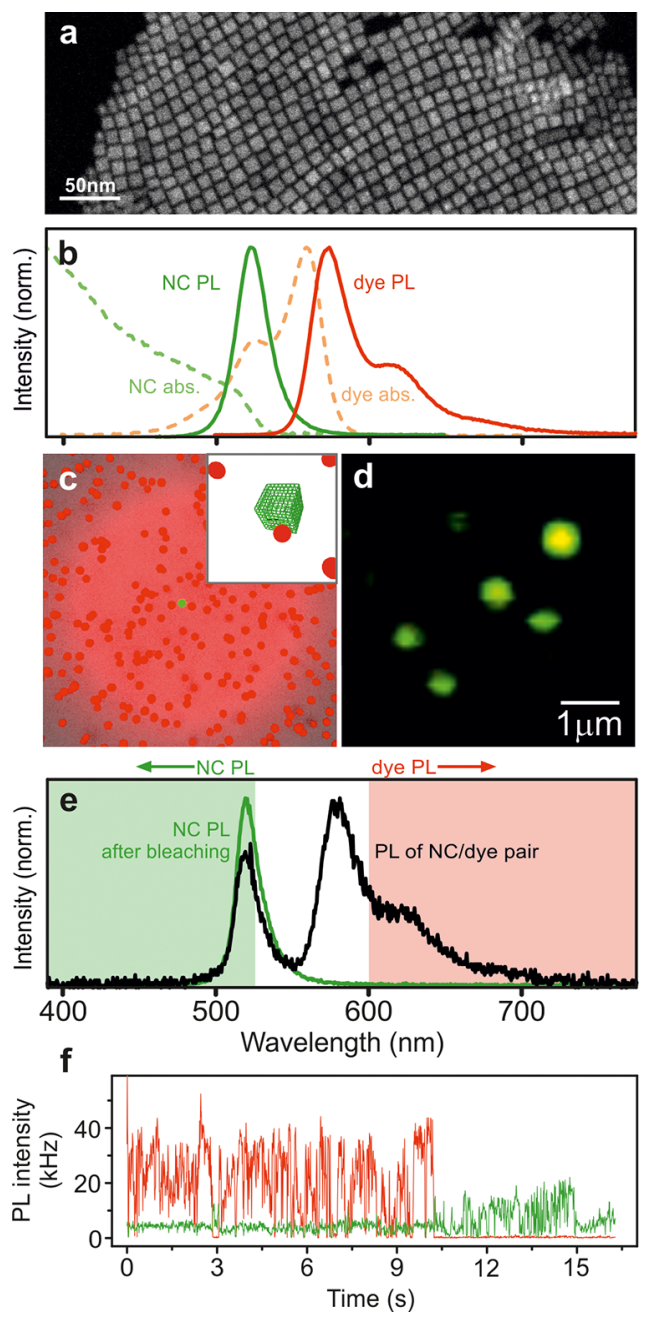

Figure 1. FRET assemblies consisting of $\mathrm{FAPbBr}_{3}$ perovskite NCs as a donor and cyanine 3 (Cy3) as an acceptor dye. (a) TEM image of the NCs. (b) Ensemble absorption and PL spectra of NCs (light and dark green curves) and $\mathrm{Cy} 3$ (orange and red curves) dispersed in toluene solution. (c) Schematic of the FRET sample with a $10 \times 10 \times$ $10 \mathrm{~nm}^{3}$ sized NC (green crystal in the center) surrounded by single dye molecules (red dots) embedded in a $100 \mathrm{~nm}$ thick PMMA film. (d) Confocal fluorescence microscopy image of a NC-dye FRET couple sample excited at $405 \mathrm{~nm}$, with the PL detected above $409 \mathrm{~nm}$. (e) Spectra of a single diffraction-limited spot, as shown in (d). The black spectrum was measured during the first $4 \mathrm{~s}$ of exposure and the green spectrum between 15 and $19 \mathrm{~s}$, after photobleaching of the dye molecule occurred. The green and red shaded areas indicate the detection channels of the PL of the NCs and dye, respectively. (f) PL intensity transient of a single NC-dye FRET pair excited at $405 \mathrm{~nm}$ and detected with the two detection channels indicated in panel (e). Photobleaching of the acceptor at $10 \mathrm{~s}$ leads to a sudden increase in donor PL.

Information in Figure S1. The acceptor dye displays a pronounced and almost digital blinking behavior and bleaches in a single step after $\sim 10 \mathrm{~s}$ (red curve). Residual PL of the NC is observed throughout the measurement (green curve), but this increases instantly once the acceptor is bleached. This observation offers a strong indication that we indeed excite only one single dye molecule by FRET from the NC. ${ }^{32}$ Timecorrelated single-photon counting (TCSPC) allows us to additionally measure the PL lifetime of the remaining $\mathrm{NC}$ emission and the PL lifetime of the dye. The PL lifetime of the 
NC decreases strongly from 13.4 to $4.1 \mathrm{~ns}$, whereas the PL lifetime of the dye increases slightly from 2.2 up to $3.5 \mathrm{~ns}$ (see Figure S2 and discussion thereof in the Supporting Information). This observation is consistent with FRET occurring from the NC to the dye molecules. In addition, we conclude that the radiative rate of the dye is not impacted by any dielectric interactions with the nearby NC.

To confirm that energy transfer occurs only between one single NC and a single dye molecule, confocal scan images were obtained at different excitation wavelengths and for different spectral detection windows. Additionally, we analyzed the photon statistics to check for signatures of photon antibunching-a fingerprint of single-particle emission-as indicated schematically in Figure $2 a{ }^{33}$ We excite the sample with laser pulses with a repetition rate of 2.5 or $5 \mathrm{MHz}$, depending on PL lifetime. A 50/50 beam splitter divides the
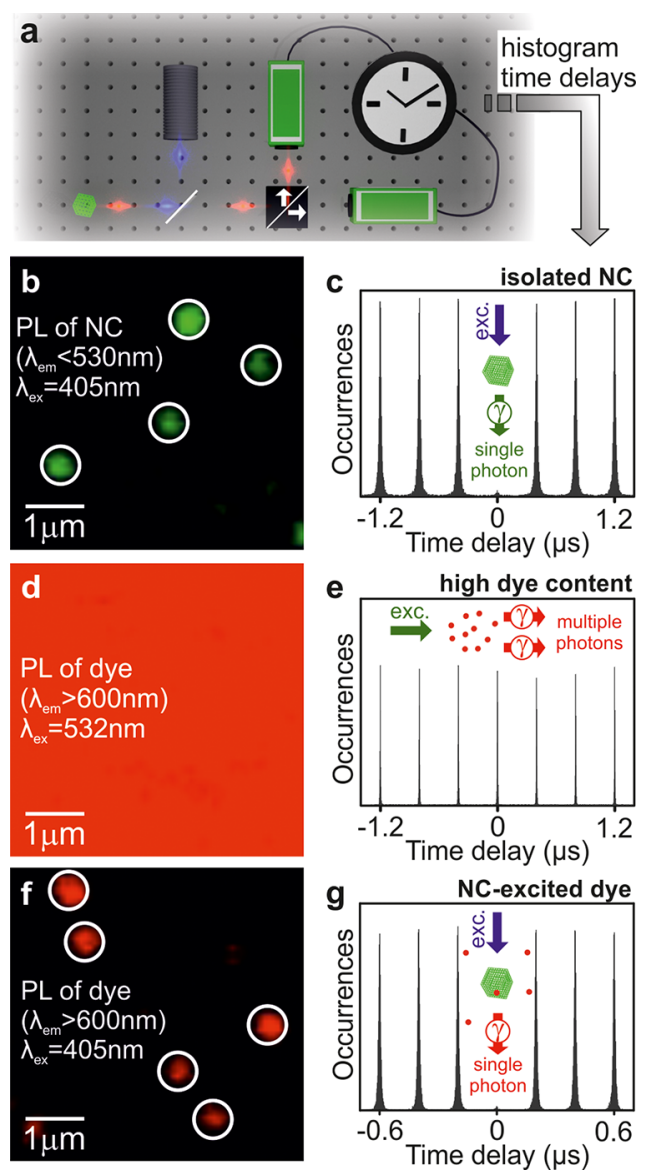

Figure 2. Fluorescence photon statistics of single NCs, dye molecules, and NC-dye FRET couples determined by second-order intensity cross-correlation. (a) Second-order cross-correlation under pulsed excitation (blue wave packets). PL (red wave packets) passes through a beam splitter and is recorded by two photodiodes. The time delay between the two detection events is measured and plotted in a histogram. (b,d,f) Confocal scanning microscope images of NCs (at single-particle concentration) and dye molecules (at a concentration 1000-fold above single-molecule concentration) embedded in PMMA. (c,e,g) Antibunching histograms for the samples shown in b, $d$, and $f$. The accumulated antibunching data of 20 NCs and 20 NC-dye FRET pairs is shown in (c) and ( $g$ ). The insets illustrate schemes for single-photon emission from the NCs (c), multiphoton emission from simultaneous excitation of multiple dye molecules (e), and single-photon emission from NC-dye FRET pairs (g). emission, which is detected on two avalanche photodiodes (APDs) and recorded by TCSPC, to yield the arrival times of each photon detected in either channel. The photon stream is analyzed with respect to recording two photons at a certain time delay between the two detection channels. These coincidence events are binned in time intervals of 100 ps and summed up in a histogram, as shown in Figure 2c,e,g, which we refer to as antibunching histograms in the following.

First, we investigate the photon statistics of the NCs to confirm that the particles used here are indeed single-photon sources. We, therefore, prepared a NC sample without dyes, excited this sample at $405 \mathrm{~nm}$, and detected the $\mathrm{PL}$ at wavelengths below $530 \mathrm{~nm}$. A confocal scan image over an area of $5 \times 5 \mu \mathrm{m}^{2}$ is shown in green in Figure $2 \mathrm{~b}$, containing four NCs. The diffraction-limited NC PL spots are marked by white circles. The NCs detected are subsequently placed individually in the excitation focus of the microscope to record the stream of emitted photons and calculate the corresponding antibunching histogram (Figure 2c). The occurrences of detecting two photons at different delay times display peaks at multiples of $400 \mathrm{~ns}$, the interval corresponding to the $2.5 \mathrm{MHz}$ excitation rate. However, only very few coincidences are detected for zero time delay, the central peak of the histogram, compared to the lateral peaks at multiples of the $400 \mathrm{~ns}$ delay time. The quality of antibunching is defined by the ratio of central coincidences to the mean number of coincidences in the lateral peaks, where a ratio of 0 corresponds to perfect single-photon emission and a ratio of 1 or $100 \%$ to a classical light source. As for previously reported perovskite $\mathrm{NCs},{ }^{34-37}$ the NCs used here evidently serve as highly deterministic single-photon sources. However, such NCs are not perfect single-photon sources, as will be discussed in the following.

Next, we repeated the experiment on a NC-dye FRET sample, exciting in the dye absorption at $532 \mathrm{~nm}$ and detecting the dye PL above $600 \mathrm{~nm}$. As shown in Figure 2d, the PL of the sample appears almost continuous over a $5 \times 5 \mu \mathrm{m}^{2}$ scan area. We again studied the photon stream of several diffractionlimited spots to construct the antibunching histogram in Figure 2e. No photon antibunching is observed under these conditions due to the large number of single dye molecules present in the diffraction-limited detection area. In contrast, exciting the same sample at $405 \mathrm{~nm}$ and again detecting the dye PL above $600 \mathrm{~nm}$ leads to the confocal scan image in Figure $2 \mathrm{f}$. The positions of the NCs are resolved clearly, as marked by white circles. Under these conditions, only the PL emission of the dye molecules is detected, which can only be excited through the NCs by FRET. The corresponding antibunching histogram in Figure $2 \mathrm{~g}$ exhibits almost perfect photon antibunching, which implies that the NCs can only excite one single dye molecule at once. Here, we measured with a repitition rate of $5 \mathrm{MHz}$, due to the shorter PL lifetime of the NC-dye FRET pairs as compared to the NCs alone. In addition, the emission polarization anisotropy underpins this conclusion because the $\mathrm{NC}$-dye couple shows highly polarized PL as opposed to the unpolarized PL observed from single NCs (see Figure S3 and discussion thereof in the Supporting Information). The unpolarized PL from single NCs together with the strong photon antibunching allows us to conclude that the transition dipole moment (TDM) of the NC must fluctuate in time.

For perovskite NCs, it is well-known that biexciton emission can occur, which diminishes the quality of deterministic singlephoton emission and is also responsible for the fact that the 

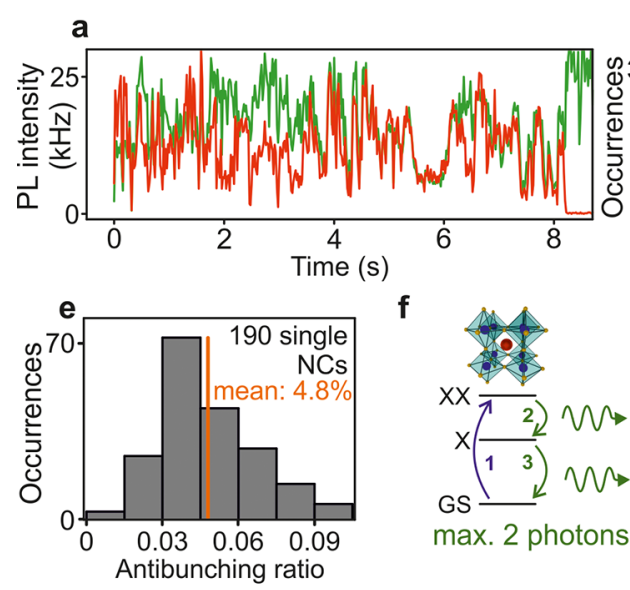

f

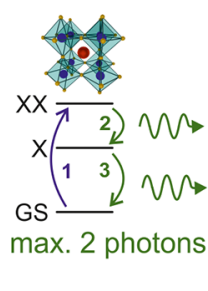

b $\quad \mathbf{C}_{\mathrm{NC}}$ photons after 2ns $\mathbf{d}$
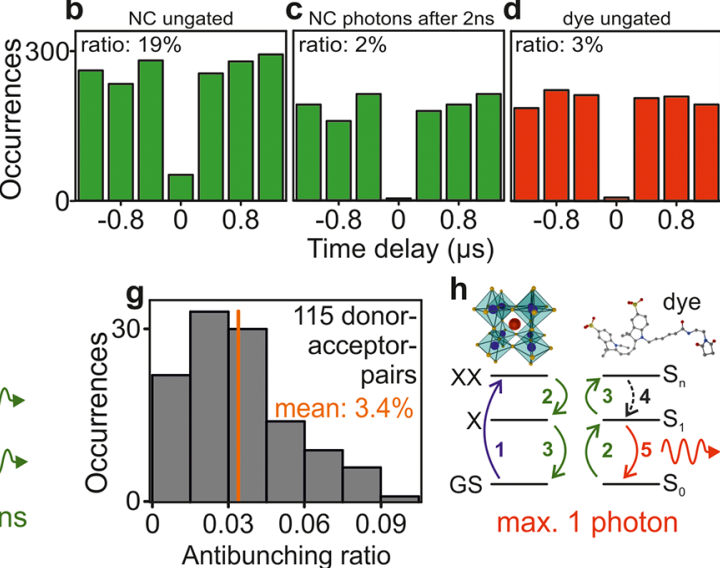

$h$

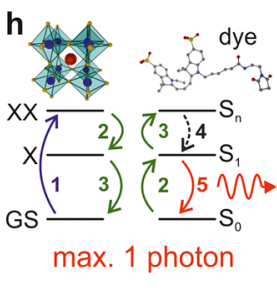

Figure 3. Comparison of the antibunching ratio from isolated NCs and NC-dye FRET assemblies. (a) PL intensity transient of a NC-dye FRET pair (green: donor channel; red: acceptor channel). The acceptor bleaches at $\sim 8 \mathrm{~s}$. (b-d) Antibunching histograms from the NC-dye FRET pair transient shown in (a) for the donor channel (green) and acceptor channel (red). Without gating out any photons, biexciton emission raises the photon coincidence peak at zero delay time (b). With gating out photons arriving within $2 \mathrm{~ns}$ after the excitation pulse, detected in the NC donor channel (c), the biexciton contribution is effectively removed, lowering the ratio to $2 \%$. Almost the same ratio is seen when considering only the dye emission of the FRET pair, without any photon gating: the FRET process to the dye acts as an effective gate to biexciton contributions. Note that these histograms, in contrast to those in Figure $2 \mathrm{c}, \mathrm{e}, \mathrm{g}$, are compiled from only one single emitter and are hence noisier. The antibunching ratio is defined as the ratio of the integrated central peak to lateral peaks. (e,g) Antibunching ratio for 190 single NCs and 115 NC-dye FRET pairs. The average ratio improves from $4.8 \%$ for single NCs to $3.4 \%$ for the FRET pairs (orange lines). (f,h) Illustrations of the biexciton emission process of single NCs (f) and the multiexciton gating process in a NC-dye couple (h).

central bar in the antibunching histograms here does not drop entirely to zero. ${ }^{36}$ The central peak area comprises approximately $5 \%$ of the integrated lateral peaks in Figure $2 c$, corresponding to an antibunching ratio of $\sim 0.05$. A photon stemming from a biexciton is emitted within a few picoseconds after excitation; therefore, by omitting detection events for the first few nanoseconds, we expect the antibunching ratio to improve. ${ }^{38}$ A PL emission decay of a single perovskite NC is shown in the Supporting Information in Figure S4, demonstrating a short decay with a $\sim 300$ ps lifetime, corresponding to the biexciton emission, ${ }^{36,38}$ followed by a long decay with a $\sim 17.5$ ns lifetime, corresponding to the subsequent exciton emission. This technique of photon gating using microtime tagging in the TCSPC is demonstrated in the Supporting Information. For applications requiring high antibunching fidelity, such photon gating in the detection is of limited utility because it dramatically reduces the overall photon count rate. Instead, we demonstrate here how the NC-dye FRET process allows for exciton gating to remove the biexciton component of the NC. A typical example of such a process is shown in Figure $3 \mathrm{a}-\mathrm{d}$. Figure $3 \mathrm{a}$ shows a PL intensity transient of a NC-dye FRET pair where the acceptor dye bleaches after $\sim 8 \mathrm{~s}$ (red curve). Fortunately, the PL intensities of the donor and acceptor are comparable for this FRET pair, which allows us to compare directly the photon statistics of the donor and acceptor. Figure $3 b-d$ displays the corresponding photon antibunching histograms in which each bar indicates all photon coincidences detected at a delay of -3 , $-2,1,0,1,2$, and 3 laser excitation pulses between the two detection channels. Because we consider only one single emitter in this case, the overall temporal resolution here is lower than that in the case of the average over several single emitters shown in Figure 2c,e,g. The residual PL stemming from the single NCs demonstrates a strong biexciton signature in the antibunching histograms. The overall antibunching ratio is found to be $\sim 19 \%$ (i.e., a dip of $81 \%$ at zero delay) when taking all emitted photons into account (Figure $3 \mathrm{~b}$ ), whereas the ratio drops down to only $2 \%$ (98\% dip) by gating out all photons with arrival times below 2 ns after the excitation pulse (Figure 3c). Crucially, however, even without photon gating the antibunching ratio of the acceptor dye emission in Figure $3 \mathrm{~d}$ yields a ratio of $3 \%$. Cross-correlation between the $\mathrm{NC}$ and dye photon channel without gating yields an antibunching ratio of $\sim 11 \%$, as shown in Figure S5b in the Supporting Information: the FRET mechanism constitutes a photon gating process in itself by removing the biexciton component. We note that, in this case, we were able to extract the corresponding antibunching histograms from one single NCdye FRET pair, demonstrating unambiguously that the antibunching ratio indeed improves when comparing FRETdriven dye emission to $\mathrm{NC}$ emission. Further, we measured the antibunching ratio for 190 single NCs and compared this with $115 \mathrm{NC}$-dye FRET pairs. These values are summarized in the histogram in Figures $3 \mathrm{e}, \mathrm{g}$ and S6. The ratio for the NCs scatters between 3 and $9 \%$ with a mean of $4.8 \pm 0.1 \%$. The schematic in Figure $3 \mathrm{f}$ illustrates the process responsible for multiphoton emission. The NCs are excited from the ground state, GS, to form a biexciton, XX (blue arrow 1), which decays radiatively into an exciton, $X$ (green arrow 2). Finally, this exciton decays to the ground state (green arrow 3 ) by emission of a second photon. Two photons can therefore be emitted following one excitation process. The probability for emission of two photons is generally quite low due to fast nonradiative Auger recombination, which is comparable to other semiconductor NCs. ${ }^{39,40}$ However, the probability is nonzero and was recently reported to be $6 \%$ for $\mathrm{CsPbX}_{3}$ quantum dots, ${ }^{36}$ similar to what we observe here for $\mathrm{FAPbBr}_{3} \mathrm{NCs}$.

Surprisingly, the antibunching ratio improves by almost $33 \%$ for the FRET pairs. When measuring the FRET pairs, we scanned the surface under green excitation and detected the fluorescence of the dye in the red emission channel. Therefore, we neglect all NCs that do not form a FRET pair with a dye. The concentration of $\mathrm{NCs}$ and dyes was adjusted to obtain $\sim 20$ FRET pairs in a $10 \times 10 \mu \mathrm{m}^{2}$ area. For the FRET pairs 
shown in Figure 3g, the values of the antibunching ratio scatter between 0 and $6 \%$, with a mean of $3.4 \pm 0.2 \%$. Two effects can explain the improvement of the fidelity of single-photon emission. First, energy transfer might not be perfectly efficient. In this scenario, the biexciton, $\mathrm{XX}$, in the $\mathrm{NC}$ decays radiatively before energy transfer to the dye can take place. Subsequently, the exciton, $\mathrm{X}$, decays and excites the dye into the first excited state, $S_{1}$, via FRET. As the dye decays back to its ground state, $S_{0}$, one photon is emitted. The resulting spectral separation of the two emitted photons leads to improved single-photon emission in the dye channel. In the second scenario, energy transfer of both the exciton and the biexciton takes place. We note that energy transfer of the biexciton is likely to be quite weak due to the biexciton's short PL lifetime of $\sim 300$ ps (see Figure S4 in the Supporting Information), but it is not impossible. Here, a plausible explanation for improved photon antibunching of the dye involves internal conversion in the dye, as illustrated in panel ( $\mathrm{h})$ : after the $\mathrm{XX}$ is generated in the NC (blue arrow 1 ), it decays to $\mathrm{X}$, simultaneously exciting the proximal dye molecule from $S_{0}$ to $S_{1}$ by FRET (green arrow 2 ). This process is followed by further excitation of the dye by FRET, together with a relaxation of $\mathrm{X}$ to the ground state (GS) of the NC, which can lead to further excitation of the dye by FRET from $S_{1}$ to a higher-lying state, $S_{n}$, indicated by the green arrow 3 in Figure $3 \mathrm{~h}$. However, emission generally does not occur from higher-lying states of molecules given strong vibrational coupling and the associated extremely efficient internal conversion described by Kasha's rule. The dye, therefore, relaxes by internal conversion to $S_{1}$ (dashed arrow 4) without emitting a photon and, finally, in the last step relaxes radiatively to $S_{0}$ (red arrow 5 ). For this reason, only one single photon can be emitted per excitation of the NC. In other words, the biexciton emission of the NC is gated out by FRET to higher-lying excited states of the single molecule, a process akin to singlet-singlet annihilation in conventional molecular FRET pairs. ${ }^{41}$ The photon emission characteristics of the dye are therefore imparted on the NC, a truly hybrid organicinorganic luminescence characteristic.

Besides the feature of multiexcitonic emission, the NC and dye differ in one further crucial respect: dye molecules are also characterized by strong intersystem crossing, which leads to shelving of the molecular excitation in the long-lived triplet state, preventing re-excitation. In contrast, in NCs, owing to the strong spin-orbit coupling, triplets can actually constitute the dominant emissive species. ${ }^{42}$ We therefore propose that the formation of multiple excitons in the NCs can lead to the population of higher-lying triplet states in the molecule by energy transfer to the absorbing $\mathrm{T}_{1}$ state of the molecule. Such a process should promote strong reverse intersystem crossing (RISC) in this class of dyes, ${ }^{43-47}$ thus imparting the nonsaturating absorption characteristics of the NC on the dye.

Triplet states are easily observable in single dye molecules as intermittencies in the PL. Figure 4a demonstrates this behavior for $\mathrm{Cy} 3$ embedded in PMMA under a nitrogen atmosphere and direct excitation at $532 \mathrm{~nm}$ and $0.1 \mathrm{~kW} / \mathrm{cm}^{2}$. A PL intensity trace, binned in steps of $10 \mathrm{~ms}$, is shown for a single molecule, which exhibits strong digital blinking between the background level and the maximum PL intensity of $\sim 20$ kcounts/s. Panel (b) shows the computed second-order autocorrelation function, $g^{(2)}(\Delta \tau)$, which is well described by a single-exponential fit (red curve) with an amplitude of $A=$ $1.4{ }^{48}$ This amplitude translates to an almost $60 \%$ probability of the dye residing in the dark triplet state. ${ }^{48}$ The blinking
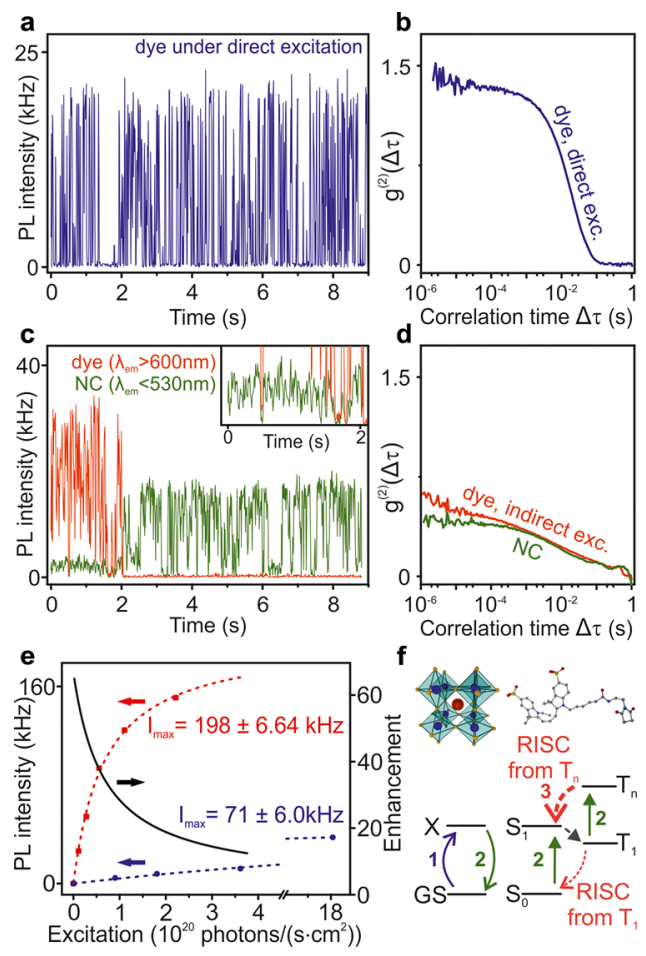

$\mathbf{f}$

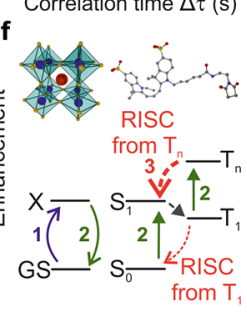

Figure 4. Comparison of the saturation behavior of single $\mathrm{Cy} 3$ dyes under direct excitation and in NC-dye FRET pairs. (a) Typical single-dye PL transient from which the fluorescence autocorrelation function $g^{(2)}(\Delta \tau)$ was computed (b). The measurements were preformed under a nitrogen atmosphere to prevent triplet quenching. (c) Typical single FRET pair PL transient, resolved in two PL detection channels, with corresponding $g^{(2)}(\Delta \tau)$ curves in (d). The correlation is plotted for dye emission under FRET excitation during the first $2 \mathrm{~s}$ of exposure (red curve) and for $\mathrm{NC}$ emission after the dye photobleaches after $2 \mathrm{~s}$ (green curve). (e) PL intensity vs excitation fluence for the dye emission under direct excitation (blue curve) and in a NC-dye FRET pair (red curve). Please note the break in the $x$ axis at a value of 4.5. The blue curve is also shown in Figure S7 without the break. Both curves can be extrapolated by a saturation function (dashed line) to yield the respective maximally achievable PL intensity, $I_{\max }$. The black curve shows the corresponding enhancement factor of the FRET pair for a given excitation intensity. (f) Illustration of the triplet deshelving reverse intersystem crossing (RISC) process, responsible for the decreased PL saturation in the FRET pair.

behavior changes drastically if the dye is excited by FRET through the NC instead of being excited directly, as demonstrated in Figure $4 \mathrm{c}$ and in the Supporting Information Figure S8a,b. In this example, a FRET couple was excited in the NC absorption at $405 \mathrm{~nm}$ and $0.1 \mathrm{~kW} / \mathrm{cm}^{2}$. The PL emission was split in half by a 50/50 beam splitter and passed into two separate Hanbury Brown-Twiss setups consisting each of a further beam splitter and two APDs. Using spectral filters, the wavelength region of the photon intensity cross-correlators were defined as $409-530 \mathrm{~nm}$ for the $\mathrm{NC}$ emission (green curve) and 600-750 nm for the Cy3 emission (red curve). For the first $2 \mathrm{~s}$ of the measurement, the dye is active and emits light. Residual luminescence is still detected in the green channel, suggesting that the energy transfer from the $\mathrm{NC}$ to the dye is not $100 \%$ efficient. After $2 \mathrm{~s}$, the dye bleaches and the emission of the NC rises. The inset plots a magnified view of the first $2 \mathrm{~s}$ of measurement, which shows that the blinking events of the NC and dye coincide. In addition, the computed autocorrelation functions $g^{(2)}(\Delta \tau)$ for the dye emission 
channel in the first $2 \mathrm{~s}$ of the measurement (red curve in panel $\mathrm{d}$, averaged over 20 spots) and the NC channel for the subsequent measurement period (green curve in panel $d$, averaged over the same 20 spots) are clearly very similar. This likeness implies that the blinking dynamics of the dye and NC are virtually identical and are only governed by the blinking of the NC. Figure S8b-d in the Supporting Information further demonstrates that this blinking is independent of the excitation intensity, which is direct proof that this blinking is not due to triplet shelving of the dye anymore, which should be dependent on the excitation intensity. The triplet blinking of the dye is reduced significantly in the $\mathrm{NC}$-dye couples, implying again that the photophysics of the NC is imparted on the dye molecule. Tian et al. showed recently that even perovskite NCs of dimensions up to $200 \times 30 \times 30 \mathrm{~nm}^{3}$ exhibit digital blinking, presumably due to chemical or structural defects that trap charged excitons and lead to nonradiative recombination. ${ }^{49}$ The same process is most probably also responsible here for the observed blinking in the $\mathrm{FAPbBr}_{3} \mathrm{NCs}$, although it is surprising to see that the single-dye emission now becomes controlled by the intermittency of the NC.

Because of the different blinking behavior of the dye molecule under direct and FRET-antenna excitation, the PL saturation characteristics must also differ. ${ }^{50}$ We prepared two samples to compare, single-molecule Cy3:PMMA and Cy3:NC:PMMA with 100-fold higher dye concentration, and acquired the average PL intensity at different excitation intensities under direct $\mathrm{Cy} 3$ excitation at $532 \mathrm{~nm}$ and under FRET pumping at $405 \mathrm{~nm}$. For each excitation intensity and sample, we acquired the average single-molecule PL intensity over approximately 250 spots. All measurements were carried out under a nitrogen atmosphere to minimize photobleaching and quenching of triplet excitations by molecular oxygen. For a better comparison between both excitation procedures, we calculated the number of photons emitted per unit time and area for each excitation wavelength. The average PL intensity of single $\mathrm{Cy} 3$ molecules under direct excitation, as plotted in Figure 4e (blue curve), saturates quickly with increasing excitation density and follows $I_{\mathrm{PL}}=I_{\max } \cdot\left[\left(I_{\mathrm{exc}} / I_{\mathrm{sat}}\right) /\left(1+I_{\mathrm{exc}} /\right.\right.$ $\left.I_{\text {sat }}\right)$ ], with $I_{\mathrm{PL}}$ the average measured PL intensity, $I_{\text {exc }}$ the excitation intensity, $I_{\text {sat }}$ the saturation intensity, and $I_{\max }$ the maximum achievable PL intensity. ${ }^{50}$ The fit yields $I_{\max }=71 \pm$ $6 \mathrm{kHz}$ in the case of direct excitation. Under indirect excitation of the dye (red curve in Figure 4e), the PL intensity follows the same saturation function but yields a 2.8 -fold increase of the maximal achievable PL intensity with $I_{\max }=198 \pm 7 \mathrm{kHz}$. Due to the almost identical blinking kinetics of the dye and the NC in the FRET pair, we conclude that this saturation stems mainly from NC blinking and not from triplet shelving in the dye. Both saturation curves (blue and red lines) yield the relative enhancement curve (black), which provides the respective enhancement factor for a given excitation intensity. For low excitation intensities, an enhancement factor greater than 50 is reached.

In Figure 4f, we propose the following mechanism for the triplet deshelving in the dye molecule: an exciton, $\mathrm{X}$, is generated in the NC (blue arrow 1), which relaxes into the ground state GS, thereby exciting the dye by FRET from $S_{0}$ into $S_{1}$ (green arrow 2). Because of intersystem crossing, the dye intermittently enters the $T_{1}$ state, which can be further excited by FRET into a $T_{n}$ state by decay of $X$ into the ground state GS (green arrow 2). The RISC process is driven by a subsequent excitation cycle of the NC. The higher excited triplet state $\mathrm{T}_{\mathrm{n}}$ can now undergo RISC into the singlet manifold, ultimately returning to the $S_{1}$ state (red arrow 3 ). ${ }^{45}$ We conclude that the $T_{1}$ state can be efficiently depopulated by RISC due to FRET from the NC to the dye.

Increasing the PL of single dye molecules is a major focus in the development of novel fluorescent probes for microscopy, with potential impact on biological and materials science research. However, for biological applications, the toxicity of lead will likely inhibit the use of perovskites, necessitating careful encapsulation approaches. Perovskite NCs constitute exquisite NC antennae to absorb light and funnel excitation energy to one single dye molecule in close proximity. Interestingly, the statistics of the emitted photons reveal that the photophysics of the acceptor dye can be exploited to gate out multiexciton emission of the NC by utilizing higher-lying excited singlet states in the dye. The same process is also responsible for removing the influence of the undesirable dark triplet state in the dye. As a consequence, saturation of the dye PL is reduced under excitation by the NC excitonic FRET antenna.

\section{ASSOCIATED CONTENT}

\section{Supporting Information}

The Supporting Information is available free of charge on the ACS Publications website at DOI: 10.1021/acs.jpclett.9b00180.

Synthesis of NCs, sample preparation, measurements of the dye PL lifetime and emission polarization in FRET pairs, and time-gated antibunching of the NC (PDF)

\section{AUTHOR INFORMATION}

\section{Corresponding Author}

*E-mail: Jan.Vogelsang@physik.uni-regensburg.de.

ORCID

Maryna I. Bodnarchuk: 0000-0001-6597-3266

Loredana Protesescu: 0000-0002-9776-9881

Maksym V. Kovalenko: 0000-0002-6396-8938

John M. Lupton: 0000-0002-7899-7598

Jan Vogelsang: 0000-0001-7952-9342

\section{Author Contributions}

The manuscript was written through contributions of all authors. All authors have given approval to the final version of the manuscript.

Notes

The authors declare no competing financial interest.

\section{ACKNOWLEDGMENTS}

The authors are indebted to the graduate training networks GRK 1570 and GRK 1626 and the collaborative research center SFB 1277 of the German Science Foundation.

\section{REFERENCES}

(1) Novotny, L.; van Hulst, N. Antennas for Light. Nat. Photonics 2011, 5, 83-90.

(2) Tam, F.; Goodrich, G. P.; Johnson, B. R.; Halas, N. J. Plasmonic Enhancement of Molecular Fluorescence. Nano Lett. 2007, 7, 496501.

(3) Rycenga, M.; Cobley, C. M.; Zeng, J.; Li, W. Y.; Moran, C. H.; Zhang, Q.; Qin, D.; Xia, Y. N. Controlling the Synthesis and Assembly of Silver Nanostructures for Plasmonic Applications. Chem. Rev. 2011, $111,3669-3712$. 
(4) Magidson, V.; Khodjakov, A. Circumventing Photodamage in Live-Cell Microscopy. In Digital Microscopy, 4th ed.; Sluder, G., Wolf, D. E., Eds.; Elsevier Academic Press Inc: San Diego, CA, 2013; Vol. 114, pp 545-560.

(5) Dixit, R.; Cyr, R. Cell Damage and Reactive Oxygen Species Production Induced by Fluorescence Microscopy: Effect on Mitosis and Guidelines for Non-invasive Fluorescence Microscopy. Plant J. 2003, 36, 280-290.

(6) Wei, Q. S.; Qi, H. F.; Luo, W.; Tseng, D.; Ki, S. J.; Wan, Z.; Gorocs, Z.; Bentolila, L. A.; Wu, T. T.; Sun, R.; et al. Fluorescent Imaging of Single Nanoparticles and Viruses on a Smart Phone. ACS Nano 2013, 7, 9147-9155.

(7) Schuler, B.; Eaton, W. A. Protein Folding Studied by Singlemolecule FRET. Curr. Opin. Struct. Biol. 2008, 18, 16-26.

(8) Acuna, G. P.; Moller, F. M.; Holzmeister, P.; Beater, S.; Lalkens, B.; Tinnefeld, P. Fluorescence Enhancement at Docking Sites of DNA-Directed Self-Assembled Nanoantennas. Science 2012, 338, 506-510.

(9) Becker, K.; Lagoudakis, P. G.; Gaefke, G.; Höger, S.; Lupton, J. M. Exciton Accumulation in Pi-conjugated Wires Encapsulated by Light-harvesting Macrocycles. Angew. Chem., Int. Ed. 2007, 46, 34503455.

(10) Soujon, D.; Becker, K.; Rogach, A. L.; Feldmann, J.; Weller, H.; Talapin, D. V.; Lupton, J. M. Time-resolved Forster Energy Transfer from Individual Semiconductor Nanoantennae to Single Dye Molecules. J. Phys. Chem. C 2007, 111, 11511-11515.

(11) Trofymchuk, K.; Reisch, A.; Didier, P.; Fras, F.; Gilliot, P.; Mely, Y.; Klymchenko, A. S. Giant Light-harvesting Nanoantenna for Single-molecule Detection in Ambient Light. Nat. Photonics 2017, 11, 657-663.

(12) Tian, Z. Y.; Yu, J. B.; Wu, C. F.; Szymanski, C.; McNeill, J. Amplified Energy Transfer in Conjugated Polymer Nanoparticle Tags and Sensors. Nanoscale 2010, 2, 1999-2011.

(13) Yeo, H.; Tanaka, K.; Chujo, Y. Effective Light-Harvesting Antennae Based on BODIPY-Tethered Cardo Polyfluorenes via Rapid Energy Transferring and Low Concentration Quenching. Macromolecules 2013, 46, 2599-2605.

(14) Adronov, A.; Frechet, J. M. J. Light-harvesting Dendrimers. Chem. Commun. 2000, 1701-1710.

(15) Yang, J.; Yoon, M. C.; Yoo, H.; Kim, P.; Kim, D. Excitation Energy Transfer in Multiporphyrin Arrays with Cyclic Architectures: Towards Artificial Light-harvesting Antenna Complexes. Chem. Soc. Rev. 2012, 41, 4808-4826.

(16) Holzmeister, P.; Pibiri, E.; Schmied, J. J.; Sen, T.; Acuna, G. P.; Tinnefeld, P. Quantum Yield and Excitation Rate of Single Molecules Close to Metallic Nanostructures. Nat. Commun. 2014, 5, 5356.

(17) Kuhn, S.; Hakanson, U.; Rogobete, L.; Sandoghdar, V. Enhancement of Single-molecule Fluorescence using a Gold Nanoparticle as an Optical Nanoantenna. Phys. Rev. Lett. 2006, 97, No. 017402.

(18) Biagioni, P.; Huang, J. S.; Hecht, B. Nanoantennas for Visible and Infrared Radiation. Rep. Prog. Phys. 2012, 75, No. 024402.

(19) Stangl, T.; Wilhelm, P.; Remmerssen, K.; Höger, S.; Vogelsang, J.; Lupton, J. M. Mesoscopic Quantum Emitters from Deterministic Aggregates of Conjugated Polymers. Proc. Natl. Acad. Sci. U. S. A. 2015, 112, E5560-E5566.

(20) Becker, K.; Lupton, J. M.; Müller, J.; Rogach, A. L.; Talapin, D. V.; Weller, H.; Feldmann, J. Electrical Control of Forster Energy Transfer. Nat. Mater. 2006, 5, 777-781.

(21) Becker, K.; Rogach, A. L.; Feldmann, J.; Talapin, D. V.; Lupton, J. M. Energetic Disorder Limits Energy Transfer in Semiconductor Nanocrystal-DNA-dye Conjugates. Appl. Phys. Lett. 2009, 95, 143101.

(22) Funston, A. M.; Jasieniak, J. J.; Mulvaney, P. Complete Quenching of CdSe Nanocrystal Photoluminescence by Single Dye Molecules. Adv. Mater. 2008, 20, 4274-4280.

(23) Harris, R. D.; Bettis Homan, S.; Kodaimati, M.; He, C.; Nepomnyashchii, A. B.; Swenson, N. K.; Lian, S. C.; Calzada, R.; Weiss, E. A. Electronic Processes within Quantum Dot-Molecule Complexes. Chem. Rev. 2016, 116, 12865-12919.
(24) Stoferle, T.; Scherf, U.; Mahrt, R. F. Energy Transfer in Hybrid Organic/Inorganic Nanocomposites. Nano Lett. 2009, 9, 453-456.

(25) Peng, H. Q.; Chen, Y. Z.; Zhao, Y.; Yang, Q. Z.; Wu, L. Z.; Tung, C. H.; Zhang, L. P.; Tong, Q. X. Artificial Light-Harvesting System Based on Multifunctional Surface-Cross-Linked Micelles. Angew. Chem., Int. Ed. 2012, 51, 2088-2092.

(26) Woller, J. G.; Hannestad, J. K.; Albinsson, B. Self-Assembled Nanoscale DNA-Porphyrin Complex for Artificial Light Harvesting. J. Am. Chem. Soc. 2013, 135, 2759-2768.

(27) Nie, S. M.; Chiu, D. T.; Zare, R. N. Probing Individual Molecules with Confocal Fluorescence Microscopy. Science 1994, 266, $1018-1021$.

(28) Protesescu, L.; Yakunin, S.; Bodnarchuk, M. I.; Bertolotti, F.; Masciocchi, N.; Guagliardi, A.; Kovalenko, M. V. Monodisperse Formamidinium Lead Bromide Nanocrystals with Bright and Stable Green Photoluminescence. J. Am. Chem. Soc. 2016, 138, 1420214205.

(29) Stangl, T.; Bange, S.; Schmitz, D.; Würsch, D.; Höger, S.; Vogelsang, J.; Lupton, J. M. Temporal Switching of Homo-FRET Pathways in Single-Chromophore Dimer Models of Pi-Conjugated Polymers. J. Am. Chem. Soc. 2013, 135, 78-81.

(30) Conroy, E. M.; Li, J. J.; Kim, H.; Algar, W. R. Self-Quenching, Dimerization, and Homo-FRET in Hetero-FRET Assemblies with Quantum Dot Donors and Multiple Dye Acceptors. J. Phys. Chem. C 2016, 120, 17817-17828.

(31) Wang, C.; Weiss, E. A. Accelerating FRET between NearInfrared Emitting Quantum Dots Using a Molecular J-Aggregate as an Exciton Bridge. Nano Lett. 2017, 17, 5666-5671.

(32) Hadar, I.; Halivni, S.; Even-Dar, N.; Faust, A.; Banin, U. Dimensionality Effects on Fluorescence Resonance Energy Transfer between Single Semiconductor Nanocrystals and Multiple Dye Acceptors. J. Phys. Chem. C 2015, 119, 3849-3856.

(33) Fleury, L.; Segura, J. M.; Zumofen, G.; Hecht, B.; Wild, U. P. Nonclassical Photon Statistics in Single-molecule Fluorescence at Room Temperature. Phys. Rev. Lett. 2000, 84, 1148-1151.

(34) Sutherland, B. R.; Sargent, E. H. Perovskite Photonic Sources. Nat. Photonics 2016, 10, 295-302.

(35) Hu, F. R.; Zhang, H. C.; Sun, C.; Yin, C. Y.; Lv, B. H.; Zhang, C. F.; Yu, W. W.; Wang, X. Y.; Zhang, Y.; Xiao, M. Superior Optical Properties of Perovskite Nanocrystals as Single Photon Emitters. ACS Nano 2015, 9, 12410-12416.

(36) Park, Y. S.; Guo, S. J.; Makarov, N. S.; Klimov, V. I. Room Temperature Single-Photon Emission from Individual Perovskite Quantum Dots. ACS Nano 2015, 9, 10386-10393.

(37) Tang, X. S.; Hu, Z. P.; Chen, W. W.; Xing, X.; Zang, Z. G.; Hu, W.; Qiu, J.; Du, J.; Leng, Y. X.; Jiang, X. F.; et al. Room Temperature Single-photon Emission and Lasing for All-inorganic Colloidal Perovskite Quantum Dots. Nano Energy 2016, 28, 462-468.

(38) Utzat, H.; Shulenberger, K. E.; Achorn, O. B.; Nasilowski, M.; Sinclair, T. S.; Bawendi, M. G. Probing Linewidths and Biexciton Quantum Yields of Single Cesium Lead Halide Nanocrystals in Solution. Nano Lett. 2017, 17, 6838-6846.

(39) Klimov, V. I.; Mikhailovsky, A. A.; McBranch, D. W.; Leatherdale, C. A.; Bawendi, M. G. Quantization of Multiparticle Auger Rates in Semiconductor Quantum Dots. Science 2000, 287, 1011-1013.

(40) Cragg, G. E.; Efros, A. L. Suppression of Auger Processes in Confined Structures. Nano Lett. 2010, 10, 313-317.

(41) Hofkens, J.; Cotlet, M.; Vosch, T.; Tinnefeld, P.; Weston, K. D.; Ego, C.; Grimsdale, A.; Müllen, K.; Beljonne, D.; Brédas, J. L.; et al. Revealing Competitive Forster-type Resonance Energy-transfer Pathways in Single Bichromophoric Molecules. Proc. Natl. Acad. Sci. U. S. A. 2003, 100, 13146-13151.

(42) Becker, M. A.; Vaxenburg, R.; Nedelcu, G.; Sercel, P. C.; Shabaev, A.; Mehl, M. J.; Michopoulos, J. G.; Lambrakos, S. G.; Bernstein, N.; Lyons, J. L.; et al. Bright Triplet Excitons in Caesium Lead Halide Perovskites. Nature 2018, 553, 189-193.

(43) Larkin, J. M.; Donaldson, W. R.; Foster, T. H.; Knox, R. S. Reverse Intersystem Crossing from a Triplet State of Rose Bengal 
Populated by Sequential 532- + 1064-nm Laser Excitation. Chem. Phys. 1999, 244, 319-330.

(44) Jia, K.; Wan, Y.; Xia, A. D.; Li, S. Y.; Gong, F. B.; Yang, G. Q. Characterization of Photoinduced Isomerization and Intersystem Crossing of the Cyanine Dye Cy3. J. Phys. Chem. A 2007, 111, 15931597.

(45) Ringemann, C.; Schönle, A.; Giske, A.; von Middendorff, C.; Hell, S. W.; Eggeling, C. Enhancing Fluorescence Brightness: Effect of Reverse Intersystem Crossing studied by Fluorescence Fluctuation Spectroscopy. ChemPhysChem 2008, 9, 612-624.

(46) Marian, C. M.; Etinski, M.; Rai-Constapel, V. Reverse Intersystem Crossing in Rhodamines by Near-Infrared Laser Excitation. J. Phys. Chem. A 2014, 118, 6985-6990.

(47) Redmond, R. W.; Kochevar, I. E.; Krieg, M.; Smith, G.; McGimpsey, W. G. Excited State Relaxation in Cyanine Dyes: A Remarkably Efficient Reverse Intersystem Crossing from Upper Triplet Levels. J. Phys. Chem. A 1997, 101, 2773-2777.

(48) Yip, W. T.; Hu, D. H.; Yu, J.; Vanden Bout, D. A.; Barbara, P. F. Classifying the Photophysical Dynamics of Single- and Multiplechromophoric Molecules by Single Molecule Spectroscopy. J. Phys. Chem. A 1998, 102, 7564-7575.

(49) Tian, Y. X.; Merdasa, A.; Peter, M.; Abdellah, M.; Zheng, K. B.; Ponseca, C. S.; Pullerits, T.; Yartsev, A.; Sundström, V.; Scheblykin, I. G. Giant Photoluminescence Blinking of Perovskite Nanocrystals Reveals Single-Trap Control of Luminescence. Nano Lett. 2015, 15, $1603-1608$.

(50) Ambrose, W. P.; Basché, T.; Moerner, W. E. Detecion and Spectroscopy of Single Pentacene Molecules in a Para-terphenyl Crystal by Means of Fluorescence Excitation. J. Chem. Phys. 1991, 95, $7150-7163$. 